\title{
Effect of sodium toluene sulfonate on the nucleation, growth and characterization of DAST single crystals
}

\author{
A.S. Haja Hameed ${ }^{\mathrm{a}}$, W.C. Yu ${ }^{\mathrm{b}}$, C.Y. Tai ${ }^{\mathrm{a}}$, C.W. Lan ${ }^{\mathrm{a}, *}$ \\ ${ }^{a}$ Department of Chemical Engineering, National Taiwan University, Taipei 10617, Taiwan \\ ${ }^{\mathrm{b}}$ Department of Molecular Science and Engineering, National Taipei University of Technology, Taipei 106, Taiwan
}

Available online 9 June 2006

\begin{abstract}
The metastable zone width and nucleation parameters such as interfacial tension were estimated for pure and sodium $p$-toluene sulfonate (NaTS)-doped 4- $N, N$ dimethylamino- $4^{\prime}-N$-methyl-stilbazolium tosylate (DAST) single crystals. Pure and additive-doped DAST single crystals were grown by the slow-cooling technique. The grown crystals were morphologically interpreted. The crystalline powders of the grown crystals were examined by X-ray diffraction to estimate the crystal lattice parameter values. From Vicker's microhardness test, the mechanical strength on $\left(\begin{array}{ll}0 & 01\end{array}\right)$ face of the crystals was found.
\end{abstract}

(C) 2006 Elsevier B.V. All rights reserved.

PACS: 81.10.Dn; 81.70.Pg

Keywords: A1. Metastable zone width; A1. Nucleation; A1. Solubility; A2. Growth from solution; A2. Recrystallization; B1. Organic compounds; B2. Nonlinear optical materials

\section{Introduction}

Ionic organic materials such as DAST (4- $N, N$ dimethylamino-4'- $N$-methyl-stilbazolium tosylate) have been investigated by many researchers for their use in optical applications. The materials have the advantages that the alignment of ionic chromophore can be controlled in a polar structure by changing the counter ion [1]. Thus, there is no need to modify the chemical structure of the chromophore itself after having determined the ionic species with large second-order hyperpolarizability. Recently, a series of stilbazolium derivatives has been studied due to their potential use for second-order harmonic generation (SHG) and electro-optic (EO) modulation [2-6]. One of the stilbazolium derivatives is DAST, which is the most useful nonlinear optical material researched to date, and which has been demonstrated to have a very large nonlinear optic (NLO) susceptibility and the largest EO coefficient $[3,7-10]$. It belongs to the monoclinic crystal

\footnotetext{
${ }^{*}$ Corresponding author. Tel./fax: + 886223633917.

E-mail address: cwlan@ntu.edu.tw (C.W. Lan).
}

system with the non-centrosymmetric space group $\mathrm{Cc}$ and the point group $\mathrm{m}$, and has the following lattice parameters: $a=10.365 \AA, b=11.322 \AA, c=17.893 \AA, Z=4$ and $\beta=$ 92.24 [3]. Duan et al. [11] found that sodium $p$-toluene sulfonate (NaTS) has a short cut-off wavelength of $280 \mathrm{~nm}$ as well as comparatively large hyperpolarizability. It is a potential chromophore for second-order NLO. The chemical structures of DAST and NaTS are shown in Fig. 1.

It is believed that NaTS is a possible dopant for DAST; both having NLO properties. NaTS is considered to dissociate in methanol generating $p$-toluene sulfonate anions and sodium cations. DAST has the anionic toluene sulfonate, which is responsible for the non-centrosymmetric nature in its own molecular structure. In the present study, we used NaTS as a dopant in the DAST solution. The nucleation behavior in the presence of NaTS in the DAST solution was investigated through induction time and metastable zone width (MZW) measurements. The pure and NaTS-doped DAST crystals were grown by the slow-cooling method. Moreover, the grown crystals were examined by X-ray diffraction analysis and Vicker's microhardness tester. 

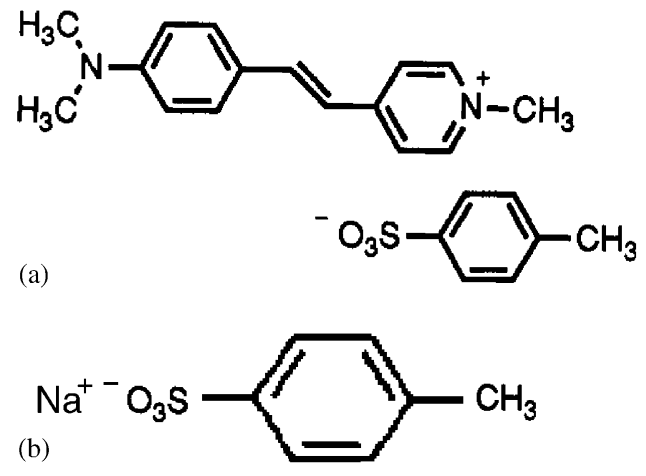

Fig. 1. Chemical structures of (a) DAST and (b) NaTS.

\section{Experimental procedure}

DAST was synthesized by the condensation of 4-methyl$N$-methyl pyridinium tosylate and $4-N, N$-dimethylaminobenzaldehyde in the presence of piperidine [12-14]. The 4-methyl- $N$-methyl pyridinium tosylate used in the condensation reaction was prepared from equimolar quantities of 4-picoline and methyl-p-toluene sulphonate. The entire synthesis process was conducted in dry nitrogen atmosphere to avoid the formation of the orange hydrated form of DAST. The resulting DAST material was further purified by recrystallization from methanol. The recrystallized DAST crystals were used for the nucleation and crystal growth experiments.

The nucleation experiments were carried out in a constant temperature bath, with a control accuracy of $\pm 0.01{ }^{\circ} \mathrm{C}$, provided with a cryostat for cooling below room temperature. Hundred milliliter of the saturated DAST solution was used in all the experiments. NaTS $(1 \mathrm{~mol} \%)$ was used in the DAST solutions. Several trials were carried out to arrive at the optimal conditions. To measure the MZW, the conventional polythermal method was employed $[15,16]$. In this method, the equilibrium-saturated solution was cooled from overheated temperature under a stirred condition until the first visible crystal was observed. Since the time required for the formation of a visible crystal after the attainment of a critical nucleus is minimal, the first crystal observed may be taken as the critical nucleus. In order to learn the induction period for the DAST solutions in different supersaturations, the conventional isothermal method was used. Here, the saturated solution was cooled to the desired temperature and maintained at that temperature, and the time taken for formation of the first crystal was measured, which gave the induction period for a selected supersaturation.

The solubility of DAST was estimated for the solvent methanol by the gravimetric method. For the growth of DAST crystals, a known amount of DAST salt was saturated in $100 \mathrm{ml}$ of methanol at $40{ }^{\circ} \mathrm{C}$. For the NaTSdoped DAST crystals, $1 \mathrm{~mol} \%$ of NaTS was used in the saturated solution. Spontaneously grown good-quality seed crystals from pure and doped DAST solutions were used to initiate the growth. The solution temperature $\left(40^{\circ} \mathrm{C}\right)$ was then reduced initially by $0.5^{\circ} \mathrm{C} /$ day in constant temperature bath for two weeks.

By means of a diffractometer (MAC Science Diffractometer MXP-3), the X-ray diffraction pattern was recorded for the powdered pure and doped DAST samples in the range $10^{\circ}-35^{\circ}$, where the monochromatic wavelength $1.5418 \AA\left(\mathrm{CuK}_{\alpha}\right)$ was used. Vickers hardness measurements were done on (001) face of the grown crystals using a microhardness tester (Model HMV-2, Shimadzu, Japan).

\section{Results and discussion}

To calculate the interfacial tension, the following theoretical consideration can be used [17]. The kinetics of nucleation depends on the thermodynamic driving force, which in turn depends on the supersaturation, temperature and impurities present in a system. The induction period, a measure of the nucleation rate is determined experimentally for the pure and NaTS-doped DAST solutions at different supersaturations. According to the classical theory of homogeneous formation of a spherical nucleus,

$\ln \tau=\ln B+16 \pi \gamma^{3} V^{2} N_{\mathrm{A}} / 3 R^{3} T^{3}(\ln S)^{2}$,

where $V$ is the molar crystal volume, $N_{\mathrm{A}}$ is Avogadro's number, $R$ is the gas constant and $S$ is the relative supersaturation $\left(S=C / C^{*}\right)$ with $C$ as the actual concentration and $C^{*}$ as the equilibrium concentration. The function $\ln B$ weakly depends on temperature and hence, there is a linear dependence between $\ln \tau$ and $(\ln S)^{-2}$ at a constant temperature. The above equation suggests a straight line for $\ln \tau$ and $(\ln S)^{-2}$ with the slope given by

$m=16 \pi \gamma^{3} V^{2} N_{\mathrm{A}} / 3 R^{3} T^{3}$.

The interfacial tension of the solid relative to this solution has been calculated from the slope of the line as

$\gamma=R T\left[3 m / 16 \pi V^{2} N_{\mathrm{A}}\right]^{1 / 3}$.

The crucial parameter involved in between a growing crystal and the surrounding mother liquor is the interfacial tension $\gamma$. In the present study, the interfacial tension was calculated using the experimentally determined induction period values. This complex parameter was determined by conducting the nucleation experiments on saturated solutions of pure and NaTS-doped DAST. From the induction period measurements, it was found that the induction period increases with the addition of NaTS.

From the induction period measurements, the interfacial tensions of pure and doped DAST were estimated and are shown in Fig. 2. The interfacial tension decreases with the addition of NaTS. The interfacial tensions of pure and doped DAST are estimated in the ranges 2.4-3.8 and $1.8-2.8 \mathrm{~mJ} / \mathrm{m}^{2}$, respectively.

The MZW of pure and NaTS-doped DAST solutions as a function of temperature is shown in Fig. 3. The results obtained from the MZW experiments show that the addition of NaTs enhances the zone width. Pure DAST 


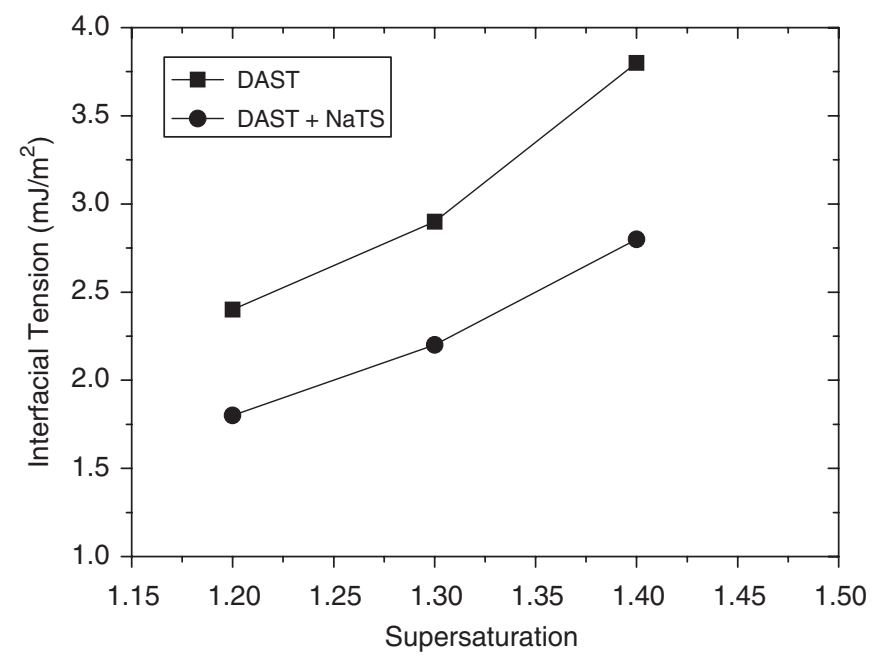

Fig. 2. Interfacial tensions of pure and NaTS-doped DAST.

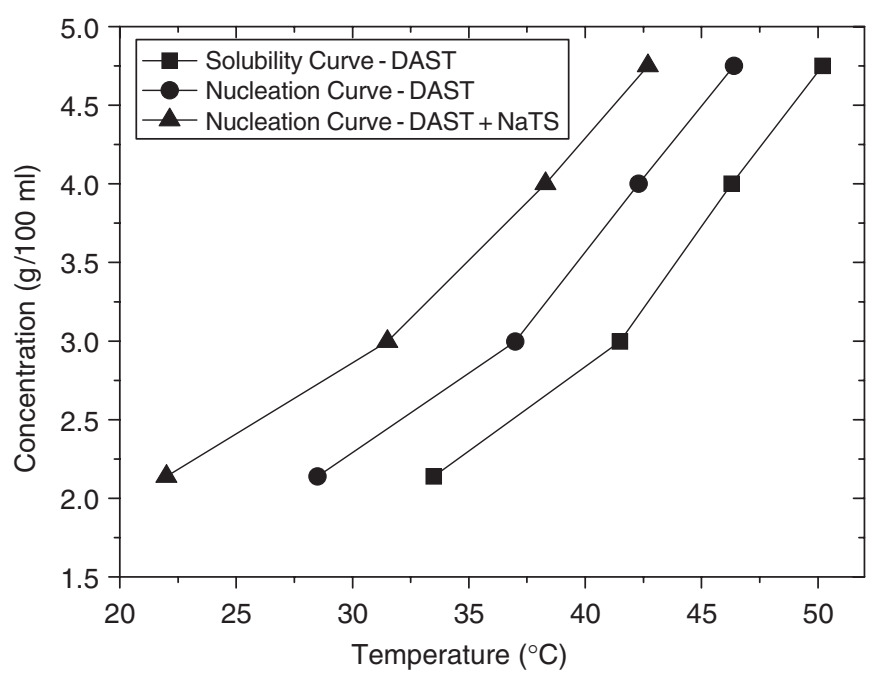

Fig. 3. Metastable zone width of pure and NaTS-doped DAST.

solution has the MZW of $5{ }^{\circ} \mathrm{C}$. The MZW of NaTS-added DAST solutions were about $11^{\circ} \mathrm{C}$. The MZW is increased by the addition of NaTS. It is believed that the polarity of the solutes in the solvent would affect the relative motion of nuclei and hence, the metastable zone. The polar solute molecules have a positive and a negative end to the molecule. If the solvent molecule is also polar, then positive ends of solvent molecules attract negative ends of solute molecules due to dipole-dipole interaction.

The grown pure and NaTS-doped DAST crystals are shown in Fig. 4. It was observed from the growth of DAST crystals that the growth rate along the $a$ and $b$ axes was the same, whereas the growth rate along the $c$-axis was very slow. The $\left\{\begin{array}{lll}0 & 0 & 1\end{array}\right\}$ habit faces grew predominantly; also, the growth was faster in both positive and negative $a$ and $b$ axes. In the grown crystal, the large $\left(\begin{array}{ll}0 & 0\end{array}\right)$ face was found and it was bounded by $\{110\},(1 \overline{1} 0)$ and small $\{020\}$ faces; the $\{020\}$ faces disappearing in the prolonged
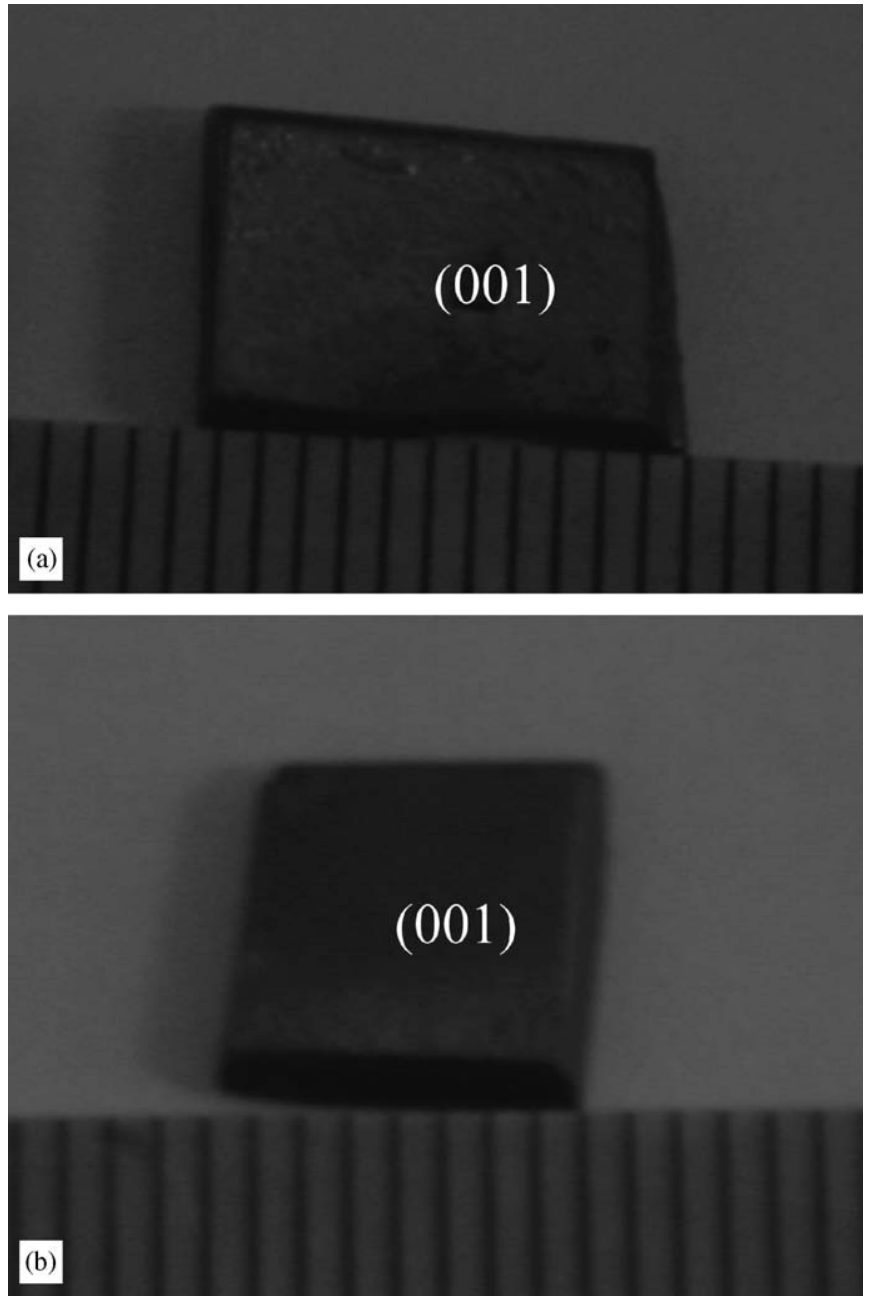

Fig. 4. Grown crystals, (a) NaTS-doped DAST $\left(11 \times 8 \times 3 \mathrm{~mm}^{3}\right)$, and (b) pure DAST $\left(8 \times 8 \times 3 \mathrm{~mm}^{3}\right)$.

growth. In the case of NaTS-doped DAST crystals, the growth rate along the $a$-axis was much larger than that in the case of the pure crystals. The morphology of the NaTSdoped DAST crystal is shown in Fig. 5(a), which resembles a rectangular shape rather than a square (as shown for the morphology of DAST crystal (Fig. 5(b)).

From the X-ray powder diffraction pattern of pure and NaTS-doped DAST samples (Fig. 6), the lattice parameter values were calculated using $2 \theta$ values of high-intensity peaks corresponding to the $h \mathrm{kl}$ planes using the monoclinic crystallographic equation. The lattice parameter values of the grown crystals are shown in Table 1. The calculated lattice parameter values of pure DAST crystals were consistent with those of the pure one and with the values reported by Marder et al. [3]. Little variation in the values of the lattice parameter was observed in the NaTSdoped DAST crystals. These values vary slightly with the values of pure DAST and this may be due to the presence of impurity in the grown crystals.

The microhardness was measured on the $\left(\begin{array}{ll}0 & 01\end{array}\right)$ face for pure and NaTS-doped DAST crystals under different 


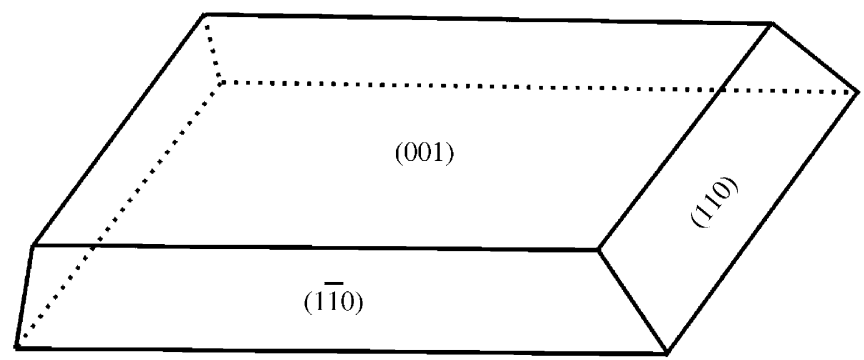

(a)

(b)

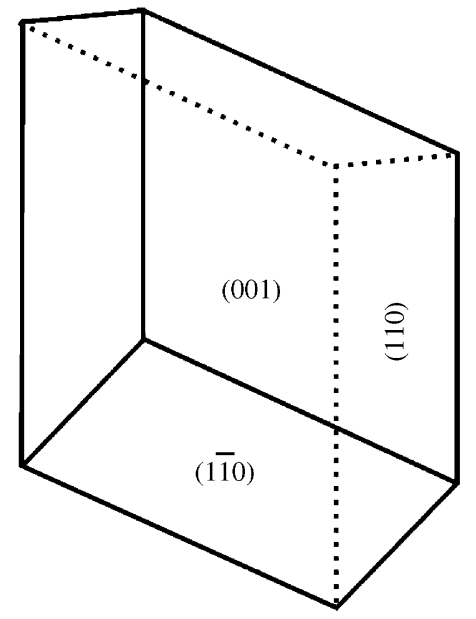

Fig. 5. Morphology of (a) NaTS-doped DAST and (b) pure DAST.

loads. The average diagonal length of the indented impression was calculated, and the Vickers microhardness number $\left(H_{\mathrm{v}}\right)$ was found from the relation $H_{\mathrm{v}}=2 P \sin (\theta / 2) /$ $d^{2}$. If the $P$ is the applied load $(\mathrm{kg})$ and $d$ is the average diagonal length of the indentation impressions $(\mathrm{mm})$, and the angle between the opposite faces of the diamond pyramid is $\theta=136^{\circ}$, then $H_{\mathrm{v}}$ in $\mathrm{kg} / \mathrm{mm}$ is given by

$H_{\mathrm{v}}=1.8544 P / d^{2}$.

The variation of microhardness $\left(H_{\mathrm{v}}\right)$ with the applied loads for pure and NaTS-doped DAST crystals is shown in Fig. 7. The microhardness number decreased with increase in the applied load. From the microhardness studies, it was revealed that the hardness of pure and doped DAST crystals was almost the same. The microhardness values of pure DAST crystals were approximately 30.5, 27.5 and $20.3 \mathrm{~kg} / \mathrm{mm}^{2}$ for the loads of 5,10 and $25 \mathrm{~g}$, respectively. The microhardness number was found in the range $19-31 \mathrm{~kg} / \mathrm{mm}^{2}$ for the doped DAST crystals.

\section{Conclusion}

In this study, the MZW of pure and NaTS-doped DAST solutions for different temperatures was determined. According to the classical theory of homogeneous formation of a spherical nucleus, the nucleation parameters were calculated. The interfacial tensions of pure and doped DAST were estimated as 2.4 and $1.8 \mathrm{~mJ} / \mathrm{m}^{2}$, respectively, at
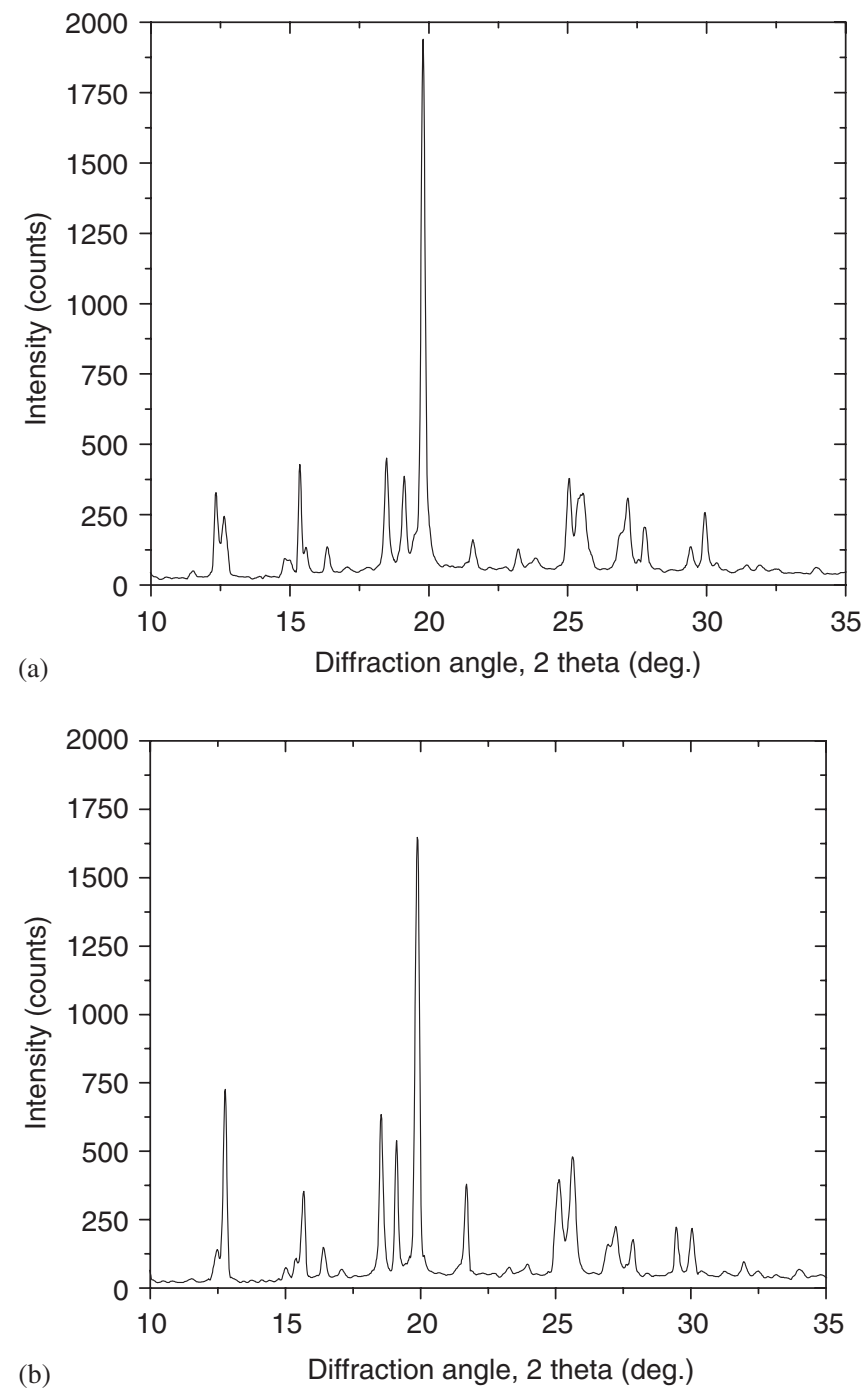

Fig. 6. X-ray diffraction pattern of (a) pure DAST and (b) NaTS-doped DAST.

Table 1

Lattice parameter values of pure and NaTS doped DAST crystals

\begin{tabular}{llll}
\hline Samples & \multicolumn{3}{l}{ Lattice parameter values $(\AA)$} \\
\cline { 2 - 4 } & $a$ & $b$ & $c$ \\
\hline Pure DAST & 10.366 & 11.323 & 17.891 \\
DAST:NaTS & 10.370 & 11.319 & 17.894 \\
\hline
\end{tabular}

lower supersaturation. Pure and NaTS-doped DAST crystals were grown by the slow-cooling method. The growth rate along the $a$-axis was found to be faster in the NaTS-doped DAST crystals as compared to pure DAST. From the X-ray powder diffraction study, the lattice parameters were determined using the simulated $h k l$ and experimentally observed $d$ values. From microhardness studies, the hardness values of the pure and doped DAST crystals were determined on the $\left(\begin{array}{ll}0 & 01\end{array}\right)$ face. It was found that the addition of NaTS to DAST did not change the 


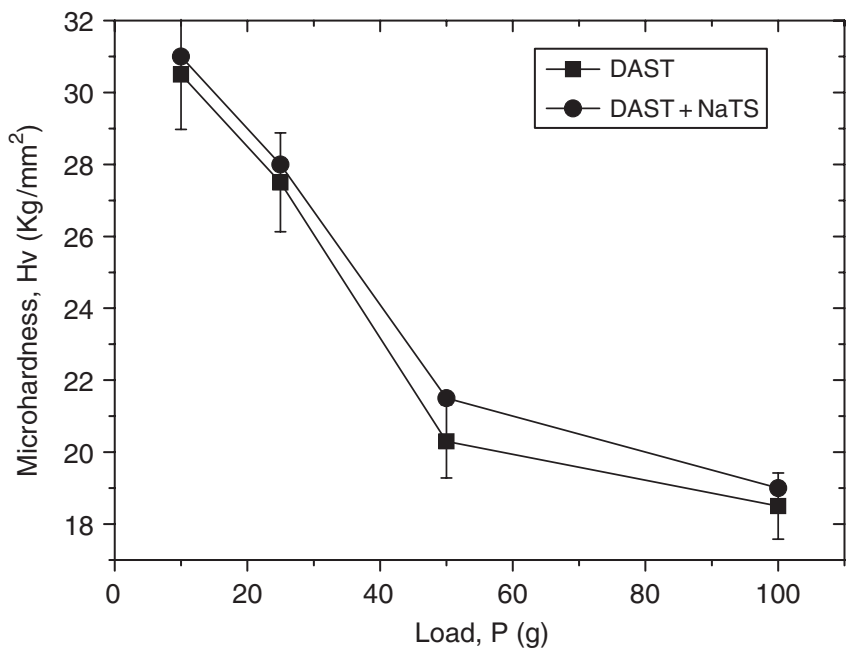

Fig. 7. Variation of microhardness with the different loads for pure and NaTS-doped DAST crystals.

hardness values as compared to the pure ones. Other characterization studies will be reported in due course.

\section{References}

[1] G.R. Meredith, Nonlinear Optical Properties of Organic and Polymeric Materials, American Chemical Society, Washington, 1983.

[2] H. Nakanishi, H. Matsuda, S.O. Okada, M. Kato, Adv. Mater. 1 (1989) 97
[3] S.R. Marder, J.W. Perry, W.P. Schaefer, Science 245 (1989) 626.

[4] S. Okada, A. Masaki, H. Matsuda, H. Nakanishi, T. Koike, T. Ohmi, N. Yoshikawa, S. Umegaki, Proc. SPIE 1337 (1990) 178.

[5] K. Sakai, N. Yoshikawa, T. Ohmi, T. Koike, S. Umegaki, S. Okada, A. Masaki, H. Matsuda, H. Nakanishi, Proc. SPIE 1337 (1990) 307.

[6] S. Okada, A. Masaki, K. Sakai, T. Ohmi, T. Koike, E. Anzai, S. Umegaki, H. Matsuda, H. Nakanishi, Nonlinear OpticsFundamentals, Materials and Devices, Elsevier, Amsterdam, 1992.

[7] C.P. Yakymyshyn, K.R. Stewart, E.P. Boden, S.R. Marder, J.W. Perry, P. Schaefer, in: Proceedings of the Second International Symposium on Organic Materials for Non-linear Optics, Royal Society of Chemistry, Cambridge, UK, 1991, p. 108.

[8] G. Knofple, R. Schlesser, R. Ducret, P. Gunter, Nonlinear Opt 9 (1995) 143.

[9] F. Pan, G. Knöfple, Ch. Bosshard, S. Follonier, R. Spreiter, M.S Wong, P. Gunter, Appl. Phys. Lett. 69 (1996) 13.

[10] Ch. Bosshard, R. Spreiter, P. Gunter, J. Opt. Soc. Am. B 18 (2001) 1620.

[11] X.M. Duan, S. Okada, H. Oikawa, H. Matsuda, H. Nakanishi, Jpn. J. Appl. Phys. 33 (1994) L1559.

[12] H. Adachi, Y. Takahashi, J. Yabuzaki, Y. Mori, T. Sasaki, J. Crystal Growth 198-199 (1999) 568.

[13] J.I. Wu, R. Gopalakrishnan, C.I.D. Tai, C.W. Lan, Jpn. J. Appl Phys. 43 (2004) 1507.

[14] A.S. Haja Hameed, W.C. Yu, Z.B. Chen, C.Y. Tai, C.W. Lan, J. Crystal Growth 282 (2005) 117.

[15] J. Nyvlt, O. Sohnel, J.J. Zola, G. Kostescky, J. Crystal Growth 62 (1983) 543

[16] N. Zaitseva, L.N. Rashkovich, S.V. Bogatyreva, J. Crystal Growth 148 (1995) 276.

[17] M. Shanmugham, F.D. Gnanam, P. Ramasamy, J. Mater. Sci. 19 (1984) 2837. 